\title{
Scalable and Automatic Dynamic Excitation of Non-Linear Structures
}

\author{
Peter Blaschke $^{1,2}$, Daniel J. Alarcón ${ }^{1}$ \\ ${ }^{1}$ Technische Hochschule Wildau, Hochschulring 1, 15745 Wildau, Germany \\ ${ }^{2}$ NV Tech GmbH, Paul-Lincke-Weg 10, 71711 Steinheim, Germany \\ peter-g.blaschke@th-wildau.de
}

\begin{abstract}
Summary:
The mathematical foundations of modal analysis assume material linearity and time invariance on a tested structure. This is, however, rarely the case in a real modal test. Complex structures often present bolted or threaded connections, or assemblies with multiple material interfaces. These connections induce non-linear force/response relationships in the structures, which hinder the application of traditional modal excitation methods. The proposed Scalable Automatic Modal Hammer (SAM) allows testing complex structures at single force/response points for a precise material characterization.
\end{abstract}

Keywords: modal testing, automatic excitation, modal analysis, modal hammer, composite

\begin{abstract}
Motivation
Experimental Modal Analysis (EMA) is the study of the dynamic properties of structures and systems in the frequency domain. The goal of EMA is to determine the resonance frequencies, mode shapes and modal damping of the studied structure. The applications of these parameters are manifold in the fields of automotive Noise, Vibration, Harshness (NVH), sound design, structural health monitoring, finite elements analysis, etc.
\end{abstract}

EMA is based on sets of equations that assume from the tested structure: 1) material linearity and homogeneity, 2) reciprocity, and, 3) time invariance [1]. These conditions are oftentimes not fully accomplished when performing modal tests in real industrial structures. For instance, bolted or screwed structures, or parts made of multiple materials such as automotive brake pads, present friction interfaces among their constructive parts. These interfaces result in non-linear modal excitation/response relationships, which are in violation of the initial EMA assumptions [2].

Traditional modal excitation techniques, such as electrodynamic shakers and handheld modal hammers, are inadequate solutions to excite these complex structures. On one hand, electrodynamic shakers require their physical attachment to the structure under study, with associated notable changes in the structure's mass and stiffness. On the other hand, handheld modal hammers are heavily operatordependent. Double impacts and a large variabil- ity in the excitation force throughout a modal test are commonplace.

\section{Scalable, Automatic Modal Hammer Excita- tion}

The solution presented in this paper profits from the advantages of hammer excitation, such as the lack of mass loading effects; while eliminating the drawbacks related to human operator variability.

The Scalable Automatic Modal Hammer (SAM) is developed to guarantee a very high degree of impact repeatability and reproducibility throughout a modal test. Furthermore, the SAM can be synchronized with a Scanning Laser Doppler Vibrometer (SLDV) for the full automatization of an impact modal test.

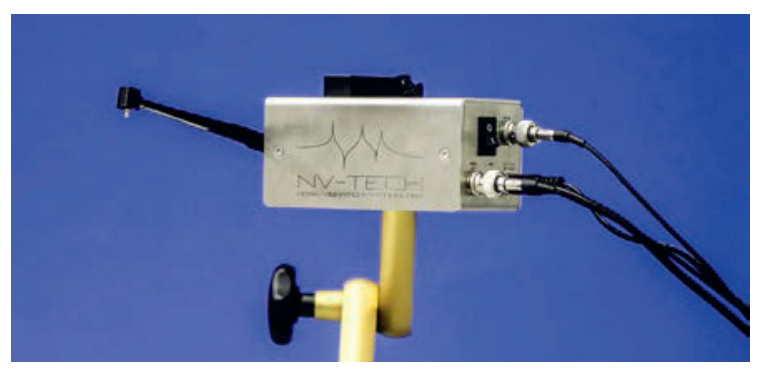

Fig. 1. The Scalable Automatic Modal Hammer (SAM).

The SAM consists of a piezoelectric impact force sensor tip built on a 3D printed ABS shaft, of proprietary topologically optimized design. The shaft is designed to be flexible enough to cause a reactionless force impulse on the structure, while keeping the sensor aligned with the structure. This assembly is driven by a stepper 
motor, controlled via USB by means of a graphical user interface. Several parameters such as hammer inwards and outwards angular velocity and acceleration can be adjusted to tailor the desired force impulse and contact time between hammer tip and structure.

\section{Repeatability Study}

A study to assess the impact repeatability and reproducibility of the SAM has been carried out in comparison with a series of manual impacts with a mini-modal hammer of the same model as that installed in the SAM, performed by an expert operator.

Three different SAM tests have been performed with objective forces $28 \mathrm{~N}, 90 \mathrm{~N}$ and $138 \mathrm{~N}$. The manual operator test was performed with an objective force of approximately 100 N. 42 impacts on a massive steel block have been recorded for each SAM test. All impacts were sampled with $250 \mathrm{kHz}$, which guarantees a proper sampling of each impact as Fig. 2 shows. Each impulse is defined with approximately 12 data points; therefore, the test results are not affected by the so-called picket-fence effect.

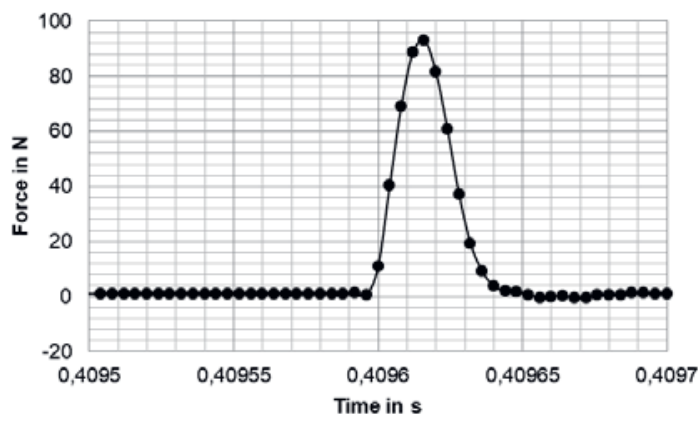

Fig. 2. $93 \mathrm{~N}$ impact sampled with $250 \mathrm{kHz}$.

Table 1 shows the mean, median and standard deviation across all performed tests. All three tests carried out with the SAM present comparable levels of standard deviation, of a maximum of $1.4 \mathrm{~N}$, and much lower values in comparison to the manual test.

Tab. 1: Mean values, median values and standard deviations for each test.

\begin{tabular}{|c|c|c|c|c|}
\hline Test & Manual & 1 & 2 & 3 \\
\hline Objective in N & 100 & 28 & 90 & 138 \\
\hline Mean & 107,371 & 27,780 & 90,404 & 138,066 \\
\hline Median & 106,800 & 27,675 & 90,080 & 138,200 \\
\hline Std. Dev & 21,803 & 1,115 & 1,131 & 1,408 \\
\hline
\end{tabular}

\section{Reproducibility Study}

The SAM was developed with the goal of exciting structures with non-linear force/response behavior at one single working point. Slight variations in the force impulses are associated to variations in the different components of a complex composite structure. The resulting frequency response functions (FRFs), are in this case different, and the modal damping of each mode cannot be estimated confidently.

Fig. 3 shows three different overlaid FRFs of a brake rotor. The SAM was used to excite the rotor three times, using the same control parameters, and therefore, obtaining the same force impulse.

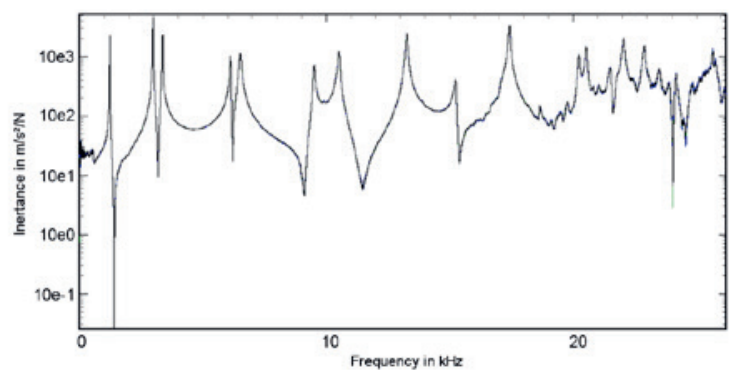

Fig. 3. Three overlaid inertance Frequency Response Functions of a brake rotor, generated with three different impacts with the SAM.

Slight differences between the black, blue and green FRFs are only observable at frequency ranges beyond $20 \mathrm{kHz}$ at the anti-resonance area at $24 \mathrm{kHz}$.

\section{Conclusions}

The main goal for modal analysis is to identify and extract the modal parameters, natural frequencies, mode shape and damping. For nonlinear structures the force input/response relationship is non-proportional. This is the reason why an extra effort has to be done to control the input force exact as possible. The work shown in this paper briefly summarizes repeatability and reproducibility studies undertaken in the SAM. The advantages of the SAM in front of a human operator are manifold, not only in regards to impact repeatability, but as well given the possibility of fully automatizing modal tests by synchronizing the SAM and a SLDV. Impact reproducibility is a necessity in this case to excite the structure during hundreds or thousands of impacts in exactly the same way.

\section{References}

[1] Irretier, H., Mathematical Foundations of Experimental Modal Analysis in Rotor Dynamics, Mechanical Systems and Signal Processing, Vol. 13, Issue 2, 183-191 (1999); doi: 10.1006/mssp. 1998.1215

[2] Blaschke, P., et al., Non-Linearity Identification of Composite Materials by Scalable Impact Modal Testing, Sensors and Instrumentation, Vol. 5, Conference Proceedings of the SEM Series, 7-14 (2017); doi: 10.1007/978-3-319-54987-3_2 